\title{
CONNECTION PROBLEMS FOR ASYMPTOTIC SERIES ${ }^{1}$
}

\author{
BY WOLFGANG WASOW
}

1. Connection problems and asymptotic power series. An analytic function is completely determined by the coefficients of its power series about one regular point, e.g. by

$$
f(z)=\sum_{r=0}^{\infty} g_{r} z^{r}
$$

The principle of analytic continuation makes it possible to calculate effectively the corresponding convergent power series about all points where a holomorphic continuation exists. However, the nature of the function near its singularities cannot be so readily deduced from the series (1.1).

Often series expansions about such singular points do exist, and sometimes it is possible to calculate them explicitly from the coefficients of the convergent expansions about a regular point. These expansions may even be power series. Nevertheless, they differ from the familiar convergent Taylor series in several decisive respects. The most important new feature is that they represent the function $f$ only in an asymptotic sense. To explain this concept, let us assume, for simplicity, that the singularity occurs at $z=\infty$. To say that the function $f$ is asymptotically represented by the series $\sum_{r=0}^{\infty} c_{r} z^{-r}$, in symbols $f(z) \sim \sum_{r=0}^{\infty} c_{r} z^{-r}$, as $z \rightarrow \infty$, means that, for all $N$, the error committed in replacing $f(z)$ by the sum of the first $N$ terms of the power series is $O\left(z^{-N}\right)$, as $z \rightarrow \infty$. Such a series may well be divergent, in fact, it usually is. If so, another important feature enters the picture: $A$ divergent asymptotic series for an analytic function at an isolated singularity never represents the function in a full deleted neighborhood, but only in certain sectors.

There exists a substantial body of theory for the "connection problem" just described, namely the problem of finding asymptotic expansions about a singular point from a given convergent expansion for the same function about a regular point. I shall not say much

An invited address delivered to the Annual Meeting of the Society in San Francisco on January 25, 1968, by invitation of the Committee to Select Hour Speakers for Annual and Summer Meetings; received by the editors April 5, 1968.

1 The preparation of this article was supported, in part, by the Office of Naval Research. 
about this work, because I am more concerned with the converse "connection problem" of finding convergent expansions at regular points from a given asymptotic expansion at a singularity.

Now, this latter problem is meaningless without some further restriction, because of another basic property of asymptotic expansions: They never determine a function uniquely. For instance, the series $0+0 \cdot z^{-1}+0 \cdot z^{-2}+\cdots$ is the asymptotic expansion, as $z \rightarrow \infty$ in the right half plane, of the function that is identically zero, as well as of $e^{-z}$.

The additional restriction imposed in this talk will be that the functions in question are solutions of a given linear homogeneous differential equation. As the solutions of such an equation form a finitedimensional vector space, the ensuing simplifications are enormous, and the ideas and methods are largely different from the theory mentioned above.

2. Local asymptotic solutions: Airy's equation. There exists a well-developed general theory that permits the asymptotic solution of linear analytic differential equations near their so-called irregular singularities (see, e.g. Wasow [1965]). Rather than to state general theorems, I shall illustrate this theory through what I consider the simplest nontrivial example, namely the equation

$$
d^{2} u / d z^{2}-z u=0,
$$

of ten called Airy's equation. Its solutions form a two-dimensional vector space over the complex numbers. This is a consequence of the basic existence and uniqueness theorems. By simple comparison of coefficients one can calculate the ascending power series for any solution that is characterized by its value and its derivative at one given point.

At $z=\infty$ the equation has an irregular singularity, and the general theory under discussion yields the following information:

(i) There exists an expression of the form

$$
z^{-1 / 4} \exp \left(\frac{2}{3} z^{3 / 2}\right) \sum_{r=0}^{\infty} a_{r} z^{-3 r / 2},
$$

where the $a_{r}$ are certain constants, which satisfies Airy's equation in the formal sense, i.e. its insertion into the left member of Airy's equation, termwise differentiation and rearrangement of terms, produces a series all of whose terms are zero. The coefficients $a_{\boldsymbol{r}}$ can be recursively calculated.

(ii) Corresponding to every sufficiently narrow sector $S$ of the z-plane 
there exist two linearly independent solutions $u_{1}(z), u_{2}(z)$ that have the series (2.2) as asymptotic expansion, as $z \rightarrow \infty$ in $S$. The determination of $z^{3 / 2}$ has to be taken different in the two series.

The asymptotic relation is to be understood in the sense that

$$
u_{j}(z) z^{1 / 4} \exp \left(-\frac{2}{3} z^{8 / 2}\right) \sim \sum_{r=0}^{\infty} a_{r}\left(z^{-3 / 2}\right)^{r}, \quad j=1,2 .
$$

The information contained in this result is local in two respects:

(i) It pertains to the limiting behavior, as $z \rightarrow \infty$. Nothing is said about the values at any finite point.

(ii) Different sectors $S$ may have different solutions $u_{j}(z)(j=1,2)$ associated with them.

Accordingly, two types of connecting problems arise.

(i) Central connecting problems. Find solutions with known expansions both at infinity and at some finite point where the solution is holomorphic.

(ii) Lateral connecting problems. For a solution with known asymptotic series, as $z \rightarrow \infty$ in one sector $S$, find expansions valid as $z \rightarrow \infty$ in other sectors with the same vertex.

It is to be expected that these two problems are closely interrelated.

The previously mentioned nonuniqueness of asymptotic series is an annoying obstacle. The concept of a "subdominant" or "recessive" solution is a great help in this connection. I shall explain it through the example of Airy's equation. Consider some subsector $S^{*}$ of $S$ in which $\operatorname{Re}\left(z^{3 / 2}\right)$ does not vanish. In such a sector one of the two solutions $u_{j}(z)$ becomes exponentially unbounded, while the other tends exponentially to zero. As all solutions are linear combinations with constant coefficients of $u_{1}$ and $u_{2}$, it is clear that the exponentially decaying solution is uniquely characterized-to within a constant factor-by this property. This remark can be extended to much more general types of linear differential equations. Following a terminology proposed by Friedrichs [1955], I shall call a solution that decays at a faster rate than all other linearly independent ones "recessive" in the sector in question. The "largest" solutions are commonly called dominant.

In the example of Airy's equation all connection problems can be solved by a procedure that is independent of the general theory and is, unfortunately, available for a very small class of very special equations only: All expansions can be derived from explicit integral representations for particular solutions, in this case from the so-called Airy integral 


$$
\operatorname{Ai}(z)=\frac{1}{2 \pi i} \int_{\Gamma} \exp \left(z t-t^{3} / 3\right) d t
$$

where $\Gamma$ is, e.g. the following path in the $t$-plane.

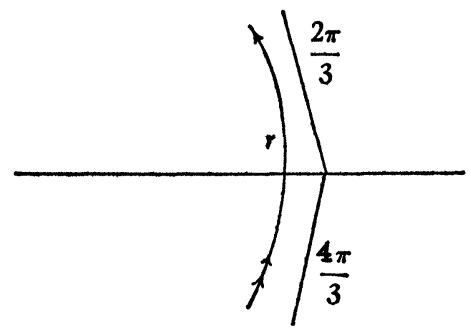

Figure 1

I shall now report, very briefly, the results that flow from an asymptotic analysis of Airy's integral, because they are simple illustrations of more general phenomena:

By the three rays $C_{j}, j=1,2,3$, of the figure below we divide the $z$-plane in to three sectors $S_{j}$.

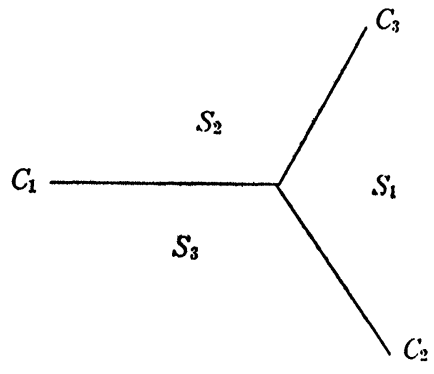

Figure 2

The direction angle of $C_{j}$ is $(2 j+1) \pi / 3$. Airy's equation possesses three particular solutions $w_{j}(z)$ that are linked by the identity

$$
w_{1}(z)+w_{2}(z)+w_{3}(z)=0 .
$$

Their power series about $z=0$ are known. As $z \rightarrow \infty$, each $w_{j}(z)$ has the asymptotic expansion (2.2) in the sector consisting of the whole plane cut along $C_{j}$. The determination of $z^{3 / 2}$ is to be so that $w_{j}$ is recessive in $S_{j}$ (and hence dominant inside the other two sectors).

The lateral connection problem for the solutions $w_{j}$ reduces in this example to the question as to their asymptotic expansions on the line $C_{j}$. This is answered by (2.4), since two of the three functions 
have expansions of the form (2.2)-with appropriate determinations for the fractional powers-on $C_{j}$. Thus, all connection problems for Airy's equation are answered.

These properties of Airy's equation are explained in many books, e.g. in Wasow [1965] and in E. T. Copson [1965]. The latter book also contains similar results for a number of classical special functions.

At the beginning of this article, I mentioned a theory which, in certain cases, permits the calculation of asymptotic series near a singular point of an analytic function from known convergent power series for that function at a regular point. As these techniques, by themselves, have nothing to do with differential equations, I will not describe them here. The earlier results are well explained in W. B. Ford [1936]. For more recent work see, e.g. H. K. Hughes [1945]. With the help of these methods Turrittin [1950] has given an almost complete analysis of the connection problems for the equation

$$
d^{n} u / d z^{n}-z^{m} u=0 .
$$

Certain systems of differential equations with linear coefficients have been globally analyzed by a similar method by Okubo [1963], [1965].

The approaches mentioned so far for the solution of connection problems might be called special function methods. When available, they yield the most complete and explicit information. Their ramifications fill many books on special analytic functions.

3. Problems with a parameter: Uniform simplification. When confronted with a complicated differential equation it is very natural to look for a simpler problem with the same essential characteristics that can be solved, and then to reduce the original problem to the simpler one by some perturbation argument. For instance, if the differential equation is of the form

$$
d^{2} u / d z^{2}-(z+h(z)) u=0
$$

where $h(z)$ is "small" with respect to $z$, as $z \rightarrow \infty$, one might expect that its solutions should asymptotically behave essentially like those of Airy's equation (2.1). However, an analytic function $h(z)$ cannot be bounded in the whole finite plane and be "smaller" than $z$ at infinity without being a constant, hence such a perturbation approach can succeed, at best, only if certain regions of the $z$-plane are excluded. Olver has written several papers (e.g. Olver [1965]) in this spirit.

There is another perturbation scheme, which is based on introducing a small parameter into the differential equation. It can be explained as follows: 
Let a new variable $t$ be defined by setting

$$
z=\epsilon^{-\alpha} t,
$$

where $\epsilon$ is a real or complex parameter and $\alpha>0$. If $t$ assumes all values in the disk $|t| \leqq t_{0}$, the old variable $z$ ranges over the whole $z$-plane, as $\epsilon \rightarrow 0$. With $\alpha=2 / 3$, which is here the most convenient choice, Airy's equation becomes

$$
\epsilon^{2}\left(d^{2} u / d t^{2}\right)-t u=0 .
$$

The asymptotic problem as to the behavior of the solutions of (3.3) in a bounded $t$-domain, as $\epsilon \rightarrow 0$, is thus exactly equivalent to the previous, parameterless problem, as $z \rightarrow \infty$, for Airy's equation and is therefore completely solved.

Now, we repeat with this new form of the equation the remark made before, that a "small" perturbation may be expected to change the asymptotic form of the solutions only slightly. A perturbed equation would now be of the form

$$
\epsilon^{2}\left(d^{2} u / d t^{2}\right)-(t+\epsilon h(t, \epsilon)) u=0,
$$

where $h(t, \epsilon)$ is holomorphic for $|t| \leqq t_{0}, 0 \leqq \epsilon \leqq \epsilon_{0}$, say.

Differential equations that depend in a singular manner on a small parameter have been widely studied, not only because of their relation to parameterless problems but also because of their independent interest in pure and applied mathematics ( $\epsilon^{2}$ may be Planck's constant or viscosity, for example).

Our particular conjecture as to the equation (3.4) has been answered in the affirmative in the most satisfactory form: the asymptotic theory of this differential equation can be completely reduced to that of equation (3.3) and, hence, to Airy's equation. This approach goes back to R. Langer (Langer [1931] and many subsequent papers). The nucleus of the technique will now be sketched in a considerably generalized version (see Wasow [1965]).

First, generality and better structural insight is gained if equation (3.3) is replaced by the equivalent two-by-two system of first order equations

$$
\epsilon(d v / d t)=A_{0}(t) v
$$

where $v$ is the vector

and

$$
v=\left(\begin{array}{c}
u \\
\epsilon d u / d t
\end{array}\right)
$$




$$
A_{0}(t)=\left(\begin{array}{ll}
0 & 1 \\
t & 0
\end{array}\right)
$$

As the perturbed system we take

$$
\epsilon(d w / d t)=A(t, \epsilon) w
$$

with

$$
A(t, \epsilon)=\sum_{r=0}^{\infty} A_{r}(t) \epsilon^{r}, \quad|t| \leqq t_{0}, \quad|\epsilon| \leqq \epsilon_{0},
$$

where all matrices $A_{r}(t)$ are holomorphic in $|t| \leqq t_{0}$. Actually, the system (3.7) is more general than it appears, for a large class of differential equations can be reduced to this form by preliminary reductions.

When speaking of transforming one differential equation into another, one must keep in mind the obvious fact that, in a sense, every ordinary linear differential equation such as (3.7) can be changed into any other,

$$
\epsilon\left(d w^{*} / d t\right)=A^{*}(t, \epsilon) w^{*}
$$

by a transformation

$$
w=P(t, \epsilon) w^{*}
$$

with some matrix function $P(t, \epsilon)$, since substitution of (3.10) into (3.7) leads to

$$
\epsilon\left(d w^{*} / d t\right)=\left[P^{-1} A P-\epsilon P^{-1}(d P / d t)\right] w^{*},
$$

which reduces to $(3.9)$ if

$$
\epsilon(d P / d t)=A P-P A^{*} .
$$

The last formula is a differential equation for $P$ which possesses analytic solutions with respect to $t$ whenever $A$ and $A^{*}$ are analytic. As (3.12) is, in general, harder to solve than the original problem (3.7) and as this remark says nothing about the dependence on $\epsilon$, it is, at best, only the first step of the work to be done.

More specific and much deeper is the following

TheoRem. The system (3.7) can be reduced to (3.5) by a transformation $w=P(t, \epsilon) v$ with a matrix $P(t, \epsilon)$ that has the following properties.

(i) $P(t, \epsilon)$ and $P^{-1}(t, \epsilon)$ are holomorphic for $|t| \leqq t_{1}, 0<\epsilon \leqq \epsilon_{1}$.

(ii) $P(t, \epsilon) \sim \sum_{r=0}^{\infty} P_{r}(t) \epsilon^{r}$ for $|t| \leqq t_{1}$, as $\epsilon \rightarrow 0+$. 
The method also yields a constructional and practicable scheme for the determination of the coefficients $P_{r}(t)$.

The long proof of this theorem falls naturally into three parts:

1. Formal reduction. The coefficients $P_{r}(t)$ are determined recursively from the condition that the transformation $w=\left(\sum_{r=0}^{\infty} P_{r}(t) \epsilon^{r}\right) v$ is to perform the reduction "formally," i.e. in the ring of formal power series.

2. Existence of analytic reductions in a sector. There exists a standard procedure for proving that a formal power series solution of a differential equation is the asymptotic expansion of a true solution. Its description here would take much too long (see Wasow [1965, $\$ \$ 26$ and 27$]$ ). In the present problem the method fails, if we wish to include the point $t=0$ into the interior of the region of validity. At best, and with considerable additional effort, the method yields the asymptotic character of the series $\sum_{r=0}^{\infty} P_{r}(t) \epsilon^{r}$, as $\epsilon \rightarrow 0+$, in certain closed sectors of the $t$-plane with vertex at $t=0$.

The exceptional role of the point $t=0$ is plausible if the special scalar case (3.4) of (3.7) is inspected. At $t=0$ the coefficient of $u$ tends itself to zero, as $\epsilon \rightarrow 0$, which is likely to affect the asymptotic structure of the solution for small $\epsilon$. The point $t=0$ is what is called a turning point for this asymptotic problem. We have to omit a precise definition of this concept. It is clear, however, that the inclusion of $t=0$ is crucial for the connection problem if we remember that we were led to the problem with a parameter by the "compression" transformation $z=\epsilon^{-2 / 3} t$. Any simply connected region of the $t$-plane which does not contain the origin has a pre-image in the $z$-plane that shrinks to the point at infinity, as $\epsilon \rightarrow 0$. In fact, it can be said that this compression transformation shifts the connection problem pertaining to expansions at $z=\infty$ into a new kind of connection problem pertaining to the neighborhood of the turning point at $t=0$. Here we can again distinguish two kinds of connection problems: central and lateral ones. The central connection problem is solved by the result for sectors, which I just mentioned. The system of sectors covers a full disk about $t=0$, but the matrices $P(t, \epsilon)$ so constructed may differ from sector to sector, even though they have the same asymptotic power series in their respective sectors. Thus, there remains still the lateral connection problem.

3. The lateral connection problem. All the sectors in which the central connection problem has been solved, as mentioned, have the origin in common. Thus, several fundamental matrix solutions for equation (3.7) are now known at that point, where they are all, in fact, asymptotically equal. One might think that it should be easy to 
find, from this information, the linear relation with constant coeffcients that must exist between any two of these solution matrices and, hence, to prove their asymptotic equivalence throughout. Actually, the necessary arguments are not quite obvious. It turns out that a solution matrix $P(t, \epsilon)$ of equation (3.12) must be chosen in a very specific way among all those that locally have the asymptotic expansion $\sum_{r=0}^{\infty} P_{r}(t) \epsilon^{r}$ in some sector if it is to serve for all sectors and, hence, for the theorem stated above. Incidentally, this section of Wasow [1965] contains an error, and I have only recently succeeded in giving a correct proof.

Asymptotic problems with a small parameter that can be reduced to Airy's equation are, of course, only a small subclass of systems of differential equations that one would like to simplify so completely to some problem with known asymptotic solutions. The literature using these or related methods has, indeed, grown to impressive size in the last thirty years. Substantial formal simplification in the ring of formal power series have been given for almost all types of second order systems of the form $\epsilon^{h}(d y / d t)=A(t, \epsilon) y$ ( $h>0$, an integer) particularly by R. Hanson and D. Russell [1967] and by Hanson [1968]. For higher order systems there are formal reductions by Wasow [1963], Hanson [1966], Kiyek [1967], Okubo [1961], and others.

Analytic solution of the central connection problem has been possible only for a much smaller subclass of these equations. The main tool is an existence theorem of Sibuya [1962] which guarantees analytic simplification when a formal reduction is available, provided certain conditions are satisfied. One of these, namely that the coefficients have to be polynomials in $t$, has been removed by Wasow [1966b], [1967]. See also Wasow [1966b] and Lee [1967].

The third part of the analysis, i.e. the solution of the lateral connection problem, has been carried out for only very few types of equations, in which solutions of the simplified equation are easily expressible in terms of Bessel or parabolic cylinder functions.

It is very tempting to formulate the following general

Conjecture. "Let $A(t, \boldsymbol{\epsilon}), B(t, \boldsymbol{\epsilon})$ be matrices holomorphic in $|t| \leqq t_{0}$, $0<\epsilon \leqq \epsilon_{0}$, and possessing uniform asymptotic power series

$$
A(t, \epsilon) \sim \sum_{r=0}^{\infty} A_{r}(t) \epsilon^{r}, \quad B(t, \epsilon) \sim \sum_{r=0}^{\infty} B_{r}(t) \epsilon^{r} .
$$

Whenever there exists a matrix power series $P=\sum_{r=0}^{\infty} P_{r}(t) \epsilon^{r}$ with coefficients holomorphic in $|t| \leqq t_{0}$ and $\operatorname{det} P_{0}(0) \neq 0$, such that $\epsilon(d P / d t)=A(t, \epsilon) P-P B(t, \epsilon)$ in the formal sense, then there exists a 
matrix $P(t, \epsilon)$ holomorphic in $|t| \leqq t_{1}, 0<\epsilon \leqq \epsilon_{1}$, such that $P(t, \epsilon)$ $\sim \sum_{r=0}^{\infty} P(t) \epsilon^{r}$ as $\epsilon \rightarrow 0+$, uniformly in $|t| \leqq t_{1}$ and that the transformation $y=P(t, \epsilon) w$ takes the differential equation $\epsilon(d y / d t)$ $=A(t, \epsilon) y$ in to $\epsilon(d w / d t)=B(t, \epsilon) w . "$

In the light of very recent unpublished work of Sibuya and Roy Lee it is unlikely that this sweeping statement is true. The situation can be described as follows: the formal series $\sum_{r=0}^{\infty} A_{r}(t) \epsilon^{r}$ and $\sum_{r=0}^{\infty} B_{r}(t) \epsilon^{r}$ define two infinite families $\phi_{A}, \phi_{B}$ of holomorphic functions, all of which have these two series as asymptotic expansions, respectively, for $\epsilon \rightarrow 0+$, uniformly in a full neighborhood of $t=0$. To every particular choice of two matrices $\tilde{A} \in \phi_{A}, \widetilde{B} \in \phi_{B}$, there do exist matrices $P(t, \epsilon)$ with the desired properties in a sufficiently narrow sector of the $t$-plane. However, for any given $\tilde{A} \in \phi_{A}$ only certain particular matrices $B^{*} \in \phi_{B}$ will have the property that the corresponding $P(t, \epsilon)$ has the formal expansion as asymptotic representations in a full neighborhood of $t=0$. The question, then, is to characterize these matrices $B^{*}$ in a usable, effective manner.

4. Problems with a parameter: Stretching and matching. When the uniform simplification method just described fails, or when it leads to an equation which, though simpler, is still not easily solved, one may try what is often called the "stretching and matching" method. Here is a simple instance of that technique:

Consider the differential equation

$$
\epsilon^{2}\left(d^{2} u / d t^{2}\right)-\left[t^{2}+\epsilon h(t, \epsilon)\right] u=0 .
$$

By the inverse of the process which produced a small parameter in the first term of Airy's equation, one can remove the small parameter from the first term in (4.1) by an appropriate "stretching" transformation $t=\epsilon^{\alpha} z$. It turns out that for $\alpha=1 / 2$ one gets, indeed,

$$
d^{2} u / d z^{2}-\left[z^{2}+h\left(\epsilon^{1 / 2} z, \epsilon\right)\right] u=0,
$$

an equation which depends analytically on the parameter $\epsilon^{1 / 2}$ and possesses, therefore, fundamental systems of solutions holomorphic with respect to $\epsilon^{1 / 2}$ at $\epsilon=0$, in any bounded $z$-domain. Such a domain corresponds to a domain in the $t$-plane that shrinks to a point, as $\epsilon \rightarrow 0$. Thus, in the $t$-plane, stretching gives results only in an "infinitesimal" neighborhood of $t=0$. The classical asymptotic theory, on the other hand, supplies us with asymptotic expansions for another fundamental system, valid in certain regions in which $|t| \geqq t_{0}>0$. Hence, for small $\epsilon$, these two regions are separated by a zone in which their validity is doubtful. We have here another version of the central connection problem. Its solution by the method under discussion 
requires two arguments: First, a proof that, at least in some possibly weaker asymptotic sense, the two types of expansions are valid representations in larger regions that do overlap for all $\epsilon$. Second, one needs a technique for finding the coefficients of the linear relations that must connect these "inner" and "outer" expansions. The first task has been tackled in papers by Nishimoto [1965], [1965b], [1966], which are based on two articles by Wasow [1961 ], [1962]. The actual connecting process has been studied more by physicists and engineers than in the mathematical literature.

If one goes beyond the example just given and its most natural generalizations, even the very first step of the stretching-matching method becomes a problem: What stretching factors should one use? This question has been answered in great generality by Iwano and Sibuya [1963] and by Iwano [1963]. It turns out that in equations with more complicated turning points at $t=0$ one has to perform more than one stretching of the form $z=t \epsilon^{-\alpha}$, each with a different value of $\alpha$. Each transformation leads to a differential equation that can be asymptotically solved in a certain "zone," and the various fundamental systems of solutions in the several zones have to be "matched" in a manner not yet quite elucidated by anybody.

In this context, still another complicating phenomenon, which I shall call "secondary turning points," has to be considered. Let me illustrate it with the simplest example, the equation

$$
\epsilon^{2}\left(d^{2} u / d t^{2}\right)-\left(t^{3}-\epsilon\right) u=0 .
$$

One indicated stretching here is

$$
t=z \epsilon^{1 / 8} .
$$

It changes the differential equation (4.2) into

$$
\epsilon^{1 / 8}\left(d^{2} u / d z^{2}\right)-\left(z^{8}-1\right) u=0
$$

which has three turning points of the Airy type at the three cube roots of unity. These correspond to $t=\epsilon^{1 / 8} \exp (2 \pi k i / 3), k=0,1,2$, and thus coalesce at $t=0$, as $\epsilon \rightarrow 0$.

If the procedure of Iwano and Sibuya is applied, a second stretching has to be performed, namely

$$
t=x \epsilon^{1 / 2},
$$

which produces the differential equation

$$
d^{2} u / d x^{2}+\left(1-\epsilon^{1 / 2} x^{3}\right) u=0,
$$

soluble by standard power series in $\epsilon^{1 / 2}$. 
Thus, even for the trivial looking differential equation (4.2), no completely adequate asymptotic analysis appears to be available. A matching procedure based on the Iwano-Sibuya theory would involve formulas connecting at least nine, probably more, fundamental systems, each known through asymptotic expansions in some region of the $(t, \epsilon)$-space.

For many problems-including equation (4.2)-this approach is probably not the simplest possible.

5. Problems with a parameter: More general asymptotic series. One underlying reason for our difficulties is our insistence, so far, that the series involved be simple power series. This is an unwarranted restriction. Any series expansion for a function such that the error involved in terminating after the $N$ th term is small, in the sense of the order of magnitude, compared to the last term retained, can be called an asymptotic expansion, and most of the properties of asymptotic power series carry over to such more general types of series.

In a sense, we have already used more general series: If in a series $\sum_{r=0}^{\infty} c_{r}(t) \epsilon^{r}$, the variable $t$ is replaced by $z \epsilon^{1 / 3}$, as in (4.3), the resulting series $\sum_{r=0}^{\infty} c_{r}\left(z \epsilon^{1 / 3}\right) \epsilon^{r}$ is no longer a power series.

Many connecting problems can be solved or, better, obviated by a skillful choice of more general asymptotic series.

Interesting results in this direction have been obtained, among others, by G. Stengle [1961], [1964]. Let me give a thumbnail sketch of his method specialized to equations of the form

$$
\epsilon^{2}\left(d^{2} u / d t^{2}\right)+g(t, \epsilon) u=0,
$$

where $g(0,0)=0$, so that $t=0$ is a turning point. We assume that $t$ and $\epsilon$ are real and $g$ is in $C^{\infty}$ for $|t| \leqq t_{0}, 0 \leqq \epsilon \leqq \epsilon_{0}$. The transformation

$$
w=\epsilon \frac{d u / d t}{u}, \quad \text { or } \quad u=\exp \left(\frac{1}{\epsilon} \int^{t} w(\tau) d \tau\right)
$$

takes (5.1) into the Riccati equation

$$
\epsilon(d w / d t)+w^{2}+g(t, \epsilon)=0 .
$$

This transformation, in the same context, has long been favored by physicists. Now, Stengle temporarily ignores the dependence of $g$ on $\epsilon$, or, in other words, he considers the two-parameter equation

$$
\epsilon(d w / d t)+w^{2}-g(t, \sigma)=0 .
$$

It can be formally satisfied by a power series of the form 


$$
w=\sum_{r=0}^{\infty} w_{r}(t, \sigma) \epsilon^{r} .
$$

Insertion of (5.5) into (5.4) and comparison of coefficients yields the recursion formulas

$$
\begin{aligned}
w_{0} & =g^{1 / 2}, \\
w_{r+1} & =-\frac{1}{2 w_{0}}\left\{\frac{d w_{r}}{d t}+\sum_{i+j=r+1 ; i, j>0} w_{i} w_{j}\right\},
\end{aligned}
$$

and, hence, two formal series corresponding to the two determinations of $g^{1 / 2}$. Of course, $\sigma$ must now again be replaced by $\epsilon$. The series are meaningless at the zeros of $g$. Nevertheless, they turn out to be useful in regions of the $(t, \epsilon)$-plane that contain points with $t=0$. It is true that at $t=0$ the coefficients $w_{r}(0, \epsilon)$ become unbounded, as $\epsilon \rightarrow 0$, but the growth is more than counterbalanced by the small factor $\epsilon^{r}$, provided certain conditions are satisfied. Essentially, these conditions amount to the requirement that no secondary turning points lie on the $t$-axis.

Stengle proves that under his assumptions the series (5.5) are, uniformly on $|t| \leqq t_{0}$, asymptotic representations of a fundamental system of two solutions of equation (5.3). The corresponding series for a fundamental system of (5.1) then are an almost immediate consequence of (5.2).

If one prefers to operate with the usual power series expansions, one can regain them from this uniform representation in $|t| \leqq t_{0}$ by reexpansion of each term with respect to whichever "stretched" variable is indicated in the region in which one is interested, followed by a rearrangement of the series.

6. Return to parameterless problems. It is true that in the recent literature much attention has been paid to asymptotic problems for differential equations depending in a singular manner on a parameter, but there have also been significant advances for the older problem of expansions as $z \rightarrow \infty$.

Here is a fruitful idea due to Hsieh and Sibuya [1966]:

Let

$$
P(z)=z^{m}+a_{1} z^{m-1}+\cdots+a_{m-1} z+a_{m} .
$$

The classical theory gives us a solution of the differential equation

$$
d^{2} u / d z^{2}-P(z) u=0
$$

that has the form 


$$
u=u_{0}(z)=\hat{n}_{0}(z) e^{Q_{0}(z)}
$$

$Q_{0}(z)$ is a polynomial in $z^{1 / 2}$, and $\mathfrak{u}_{0}(z)$ has (except for a simple factor) an asymptotic expansion in powers of $z^{-1 / 2}$, valid in the sector $|\arg z|<3 \pi /(m+2)$. The determination of $z^{1 / 2}$ is to be such that the solution $u_{0}(z)$ is recessive in the middle third of that sector. By simple symmetry arguments this result produces $m+1$ additional asymptotically known solutions $u_{1}, \cdots, u_{m+1}$ so that $u_{j}$ has a known asymptotic expansion in the sector

$$
|\arg (z+(2 j /(m+2)) \pi)| \leqq 3 \pi /(m+2)
$$

and is recessive in the middle third.

To these facts, Sibuya and Hsieh add an important new element: They prove that the solutions $u_{j}$ are entire functions of the coefficients $a_{1}, a_{2}, \cdots, a_{m}$ of $P(z)$. Hence, there must exist an expansion

$$
u_{0}\left(z, a_{1}, \cdots, a_{m}\right)=\sum_{p_{1}, \cdots, p_{n}} \mu_{p_{1}, p_{2}}, \cdots, p_{n}(z) a_{1}^{p_{1}} a_{2}^{p_{2}} \cdots a_{n}^{p_{n}} .
$$

By formal insertion of this series into the differential equation (6.2) and comparison of coefficients, one finds for each $\mu_{p_{1} p_{2} \cdots p_{n}}(z)$ an auxiliary differential equation of second order that can be solved in terms of Bessel functions. The correct boundary condition at each step can be derived from the required behavior of $u_{0}$ at infinity, which is known from the asymptotic formula (6.3). Thus, the central connection problem is completely solved.

In addition to such truly parameterless problems, there exist many papers which can be best understood by introducing a parameter, even though the authors do not do it explicitly in their notation. This is particularly true of the literature close to applications in physics, such as the papers by Olver [1965] and N. and P. Fröman [1965].

The work of the Frömans centers about differential equations

$$
d^{2} u / d t^{2}+f(t) u=0,
$$

when $f(t)$ is "large." Instead of committing themselves as to the precise meaning of that epithet they aim at strict inequalities for the solutions that remain meaningful under fairly mild conditions, but which are of interest only if certain quantities in them are much larger than the others.

They begin their analysis with the well known transformation

$$
u=f^{-1 / 4}(t) v, \quad z=\int^{t} f^{1 / 2}(\tau) d \tau
$$


which takes (6.5) into

$$
d^{2} v / d z^{2}+(1+p(z)) v=0,
$$

where

$$
p(z)=f^{-3 / 4}(t)\left(d^{2} / d t^{2}\right)\left[f^{-1 / 4}(t)\right] .
$$

In many cases $p(z)$ will be "small" as a consequence of $f(t)$ being "large." Observe that the relation between $t$ and $z$ is a kind of generalized stretching transformation: If $f(t)$ is large for moderate values of $t$, then $z$, the new independent variable, will be large.

Thus, in some approximate sense the solutions of (6.7) can be expected to be close to those of $d^{2} v / d z^{2}+v=0$. This motivates the next step which is to replace (6.7) by an equivalent 2 by 2 system, and to diagonalize the leading term by a linear transformation with constant coefficients. The leading part of the resulting system has the fundamental solution matrix

$$
\left[\begin{array}{cr}
e^{i z} & 0 \\
0 & e^{-i z}
\end{array}\right]
$$

This suggests writing the solution of the system in the form

$$
\left[\begin{array}{cr}
e^{i z} & 0 \\
0 & e^{-i z}
\end{array}\right] F(z)
$$

For the new unknown matrix $F$ one thus obtains the differential equation

$$
\frac{d F}{d z}=\frac{i}{2} p(z)\left[\begin{array}{ll}
1 & e^{-2 i z} \\
-e^{2 i z} & -1
\end{array}\right] F,
$$

which is the starting point of the subsequent work. Observe that even though $p(z)$ may be "small" in the applications, the exponentials $e^{ \pm 2 i z}$ can be very large for large $z$ in the complex plane.

Nevertheless, the Neumann series, i.e. the series obtained for the solution $F$ by systematic Picard iteration, converges whenever $p(z)$ is holomorphic. The main difference between the classical asymptotic theory and the approach of the Frommans (and of Olver) is that the latter use this Neumann series for explicit appraisals of the solution and not only as a tool for existence proofs. By means of a very detailed analysis of the series, they arrive at a set of useful inequalities that enable them to solve lateral connection problems of considerable complexity, even in domains that contain several zeros of $f$, i.e. singularities of $p$. Those points correspond precisely to the turning points 
of the theory with a small parameter. This fact can be brought out more clearly if the problem is reformulated in a slightly different form by setting

$$
f(t)=\epsilon^{-1} g(t) .
$$

7. Connection problems with several turning points. Physicists of ten run into problems where a differential equation with a parameter possesses a whole string of turning points, and the connecting problem consists in continuing asymptotic evaluations for a particular solution past, if not through, all these turning points. The methods of the Frömans and of Olver might be used to cope with such problems. Recently, more systematic attacks have been undertaken by Fedoryuk (Evgrafov and Fedoryuk [1966]) and by Sibuya [1967].

It should surprise nobody, after what I have said, that all work on such connecting problems is exceedingly involved and almost impossible even to sketch in a few sentences. I shall try, nevertheless, to give some idea of the work of Fedoryuk. The differential equation under consideration is again

$$
\epsilon^{2}\left(d^{2} u / d t^{2}\right)-P(t) u=0,
$$

where $P(t)$ is an entire function subject to certain conditions too complicated to enumerate. All these conditions are trivially satisfied when $P(t)$ is a polynomial. In this brief account I shall restrict myself to the polynomial case.

$(\alpha)$ The topology of the Stokes curves. On the basis of well-known aspects of the asymptotic theory (e.g. formulas (5.2), (5.5) and (5.6), or else formulas (6.6), (6.10)), it is plausible that the functions

$$
\exp \left\{\frac{1}{\epsilon} \int P^{1 / 2}(t) d t\right\}
$$

must figure prominently in every global asymptotic analysis of the solutions. If we limit $\epsilon$ to positive values, the level lines $\operatorname{Re} \xi\left(t_{0}, t\right)$ $=$ const. of the algebraic integral

$$
\xi\left(t_{0}, t\right)=\int_{t_{0}}^{t} P^{1 / 2}(\tau) d \tau
$$

take on a special importance. This family of curves does not depend on the initial value $t_{0}$, the choice of path in the complex $\tau$-plane or the determination of the square root in the definition of $\xi\left(t_{0}, t\right)$. Of particular interest are those curves of the form $\operatorname{Re} \xi=$ const. which pass 
through the branch points of $\xi\left(t_{0}, t\right)$, i.e. through the zeros of $P(t)$. The zeros of $P(t)$ are commonly called turning points of the differential equation, and the curves $\operatorname{Re} \xi=$ const. that contain turning points are often referred to as the Stokes curves of the problem. Evgrafov and Fedoryuk [1966] give an analysis of the graphs (they are even trees) formed by the system of Stokes curves for such differential equations. These curves divide the $t$-plane into a finite number of simply connected unbounded regions. Let us call them Stokes regions, for want of a better name. The Figures 3 and 4 , below, show two examples of such systems of curves. The multivalued function $\xi(t)$ maps the $t$ plane into a fairly complicated Riemann surface. Its analysis can be circumvented by concentrating on the images of the Stokes regions under some branch of $\xi\left(t_{0}, t\right)$. As each Stokes curve is mapped onto a straight segment or ray parallel to the imaginary $\xi$-axis, these images are vertical strips that may, in particular, be half planes. If a branch of $\xi\left(t_{0}, t\right)$ is continued analytically through an appropriate number of adjacent Stokes regions, the image of the union of these Stokes regions, and of those Stokes curves that separate two such regions, consists of the whole plane with some vertical straight cuts removed.

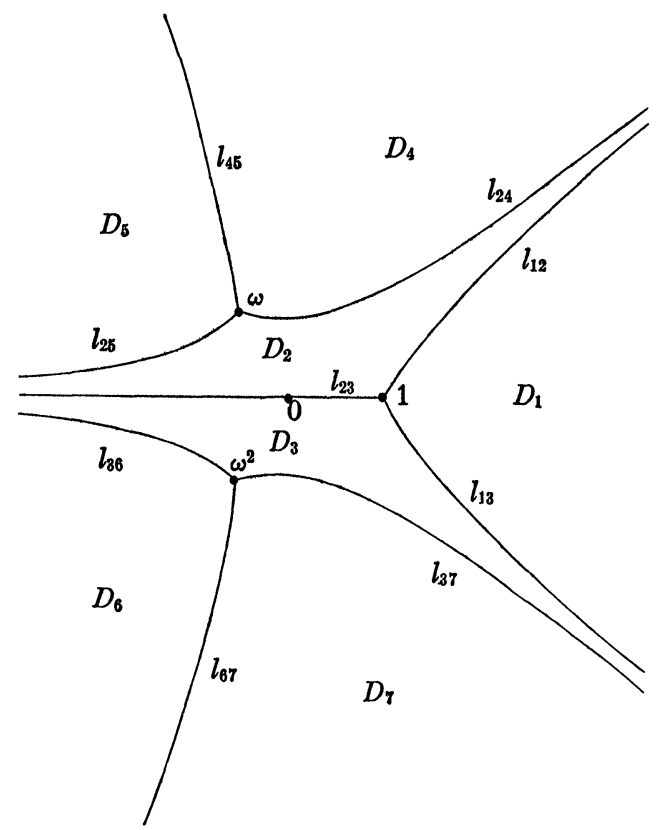

FIGURE 3. Turning points and Stokes curves for $P(t)=t^{3}-1$. 


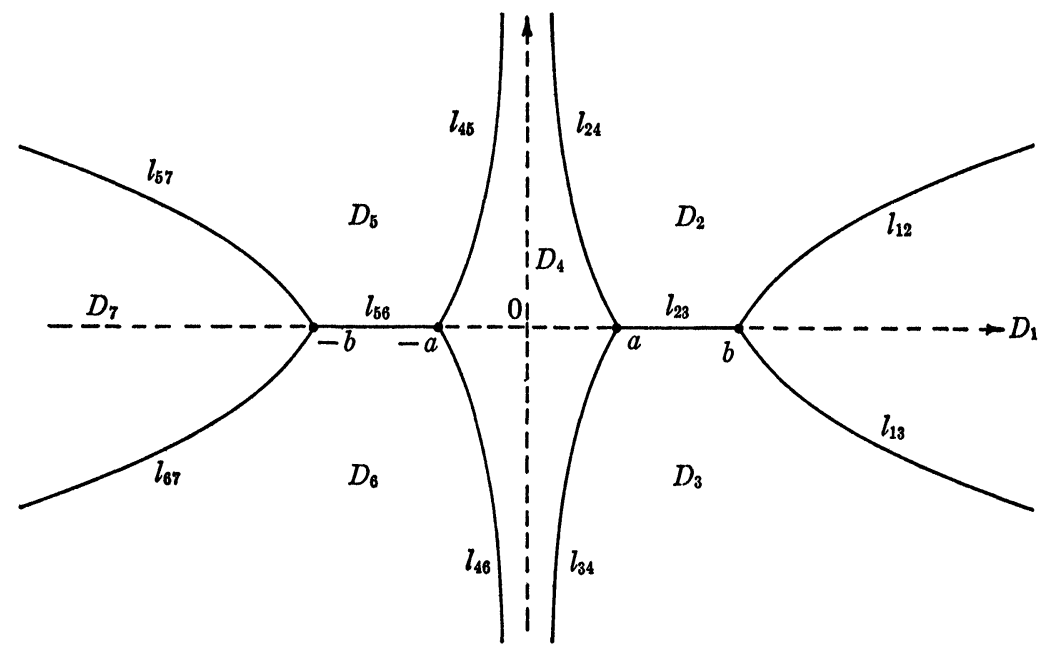

FIGURE 4. Turning points and Stokes curves for $P(t)=\left(t^{2}-a^{2}\right)\left(t^{2}-b^{2}\right)$.

Such a union of Stokes regions is called a canonical region by Fedoryuk. Illustrations of some of these mappings are given in Figures 5 and 6.

$(\beta)$ Elementary fundamental systems of solutions. There exist two standard types of asymptotic expansions for solutions of the differential equation (7.1): one that deals with the passage to the limit as $z \rightarrow \infty$, and one concerned with the behavior as $\epsilon \rightarrow 0$. By extending an approach due to G. D. Birkhoff [1933], Fedoryuk succeeds in combining these two results by proving, roughly stated, the following result: Let $R$ be a canonical region and let $\xi\left(t_{0}, t\right)$ be a branch of the function $\int_{t_{0}}^{t} P^{1 / 2}(\tau) d \tau$ that is holomorphic in $R$. Then the differential equation (7.1) has a fundamental system of solutions $u(t), v(t)$, such that

$$
\begin{aligned}
& u(t, \epsilon)=P^{-1 / 4}(t) \exp \left((1 / \epsilon) \xi\left(t_{0}, t\right)\right)[1+\mu(t, \epsilon)], \\
& v(t, \epsilon)=P^{-1 / 4}(t) \exp \left(-(1 / \epsilon) \xi\left(t_{0}, t\right)\right)[1+\nu(t, \epsilon)],
\end{aligned}
$$

where $\mu(t, \epsilon), \nu(t, \epsilon)$ are $O(\epsilon)$, as $\epsilon \rightarrow 0+$ and $o(1)$ as $t \rightarrow \infty$ in $R$. This is true uniformly for $0<\epsilon \leqq \epsilon_{0}$ and for $t$ in a subset of $R$ obtained by removing neighborhoods of the turning points and of the bounding Stokes curves.

Observe that, in spite of its strength in many respects, this theorem does not solve the central connecting problems with respect to $\epsilon$, since the neighborhoods of the turning points have to be omitted.

The fundamental system $u(t), v(t)$ so obtained depends on $R$, the 


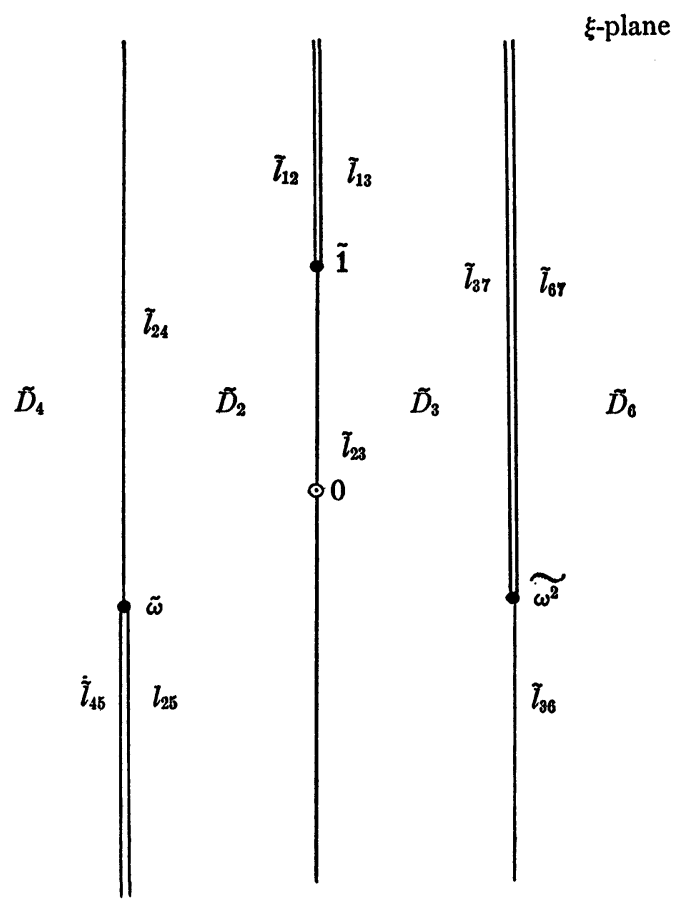

FIGURE 5. Image in the $\xi$-plane of the canonical region $D_{4} \cup D_{2} \cup D_{3} \cup D_{6}$ for $P(t)=t^{3}-1$.

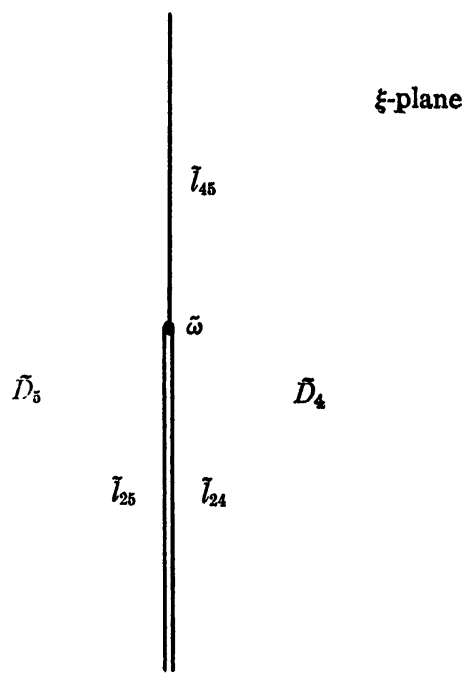

Figure 6. Image in the $\xi$-plane of the canonical region $D_{4} \cup D_{b}$ for $P(t)=t^{3}-1$. 
choice of $t_{0}$, the branch of $\xi\left(t_{0}, t\right)$ and on the determination of the branch of $P^{-1 / 4}(t)$. A precise characterisation of solutions can be given as follows: for a given canonical region $R$ let $t_{0}$ be a turning point on the boundary of $R$, let $l$ be a particular Stokes arc issuing from $t_{0}$ into the interior of $R$ (such Stokes arcs always exist). Choose the branch of $\xi\left(t_{0}, t\right)$ so that $\operatorname{Im} \xi\left(t_{0}, t\right)$ increases along $l$ in the direction away from $t_{0}$. Instead of describing directly a particular branch of $P^{-1 / 4}(t)$ we replace $u, v$ by $c u, c v$, where $c=e^{-i \alpha}$,

$$
\alpha=\lim _{t \rightarrow t_{0} ; t \in l} \arg P^{-1 / 4}(t) .
$$

Then $c u, c v$ remain the same if the branch of $P^{-1 / 4}(t)$ is changed. The particular solutions so described are called elementary fundamental systems by Evgrafov and Fedoryuk and designated by the symbol $\left(R, t_{0}, l\right)$. They are uniquely determined because each solution is recessive with respect to the passage to the limit as $t \rightarrow \infty$ in certain directions in $R$.

$(\gamma)$ The connecting matrices. Let us use the abbreviation $w\left(R, t_{0}, l\right)$ for the vector

$$
\left(\begin{array}{l}
u \\
v
\end{array}\right)
$$

formed by the unique fundamental system $u, v$ which corresponds, as explained above, to a canonical region $R$, a turning point $t_{0}$ and a Stokes arc $l$. Any two elementary fundamental systems are connected by a linear relation

$$
w\left(R_{2}, t_{02}, l_{2}\right)=\Omega_{21} w\left(R_{1}, t_{01}, l_{1}\right)
$$

where $\Omega_{21}$ is a nonsingular two-by-two matrix with constant coefficients that may-and generally will-depend on $\epsilon$. These so-called transition matrices form a finite group that can be generated from a smaller number of elementary transition matrices for which the canonical regions $R_{1}, R_{2}$ overlap or are identical.

The calculation of the elementary transition matrices offers genuine difficulties only when the two canonical regions are not identical. The central problem appears to be the transition from $w\left(R_{1}, t_{0}, l_{1}\right)$ to $w\left(R_{2}, t_{0}, l_{2}\right)$. Fedoryuk succeeds in deriving satisfactory asymptotic formulas for the corresponding elementary transition matrices when $t_{0}$ is a simple turning point, i.e. a simple zero of $P(t)$.

The Figures 3, 5, and 6 exhibit an illustration of these concepts when $P(t)=t^{3}-1$ : With a notation that should be clear from Figure 3 we can set 


$$
\begin{aligned}
& R_{1}=D_{4} \cup D_{2} \cup D_{3} \cup D_{6} \cup l_{24} \cup l_{23} \cup l_{36}, \\
& R_{2}=D_{4} \cup D_{5} \cup l_{45}, \\
& t_{0}=\omega, \\
& l_{1}=l_{24}, \quad l_{2}=l_{45} .
\end{aligned}
$$

Then the passage from $w\left(R_{1}, \omega, l_{24}\right)$ to $w\left(R_{2}, \omega, l_{45}\right)$ is one of the elementary transitions, since the turning point is the same and the canonical regions have the Stokes region $D_{4}$ in common. See Evgrafov and Fedoryuk [1966] for the-rather involved-details.

( $\delta$ ) Eigenvalue problems. Having solved the elementary connection problems, Fedoryuk has at his disposal the tools for an answer to some of the eigenvalue problems that are at the root of the physicists' interest in such mathematical theories.

Consider, e.g., the case that

$$
P(t)=\prod_{j=1}^{2 k}\left(t-a_{j}\right), \quad a_{j}<a_{j+1},
$$

and that one wants to solve the real eigenvalue problem

$$
d^{2} u / d t^{2}-\lambda^{2} P(t) u=0, \quad u \in L_{2}(-\infty, \infty) .
$$

For large real values of the parameter $\lambda$ the solutions of the differential equation can be approximately evaluated by their asymptotic expansions. The solution that is recessive, as $t \rightarrow+\infty$, will in general not be recessive as $t \rightarrow-\infty$. The asymptotic form of this solution for large negative values of $t$ can be found by continuing along the real axis and around the turning points. This amounts to the multiplication of the several corresponding elementary transition matrices. The condition that the solution be recessive for $t \rightarrow-\infty$, as well, expresses itself then as an equation for an entry of the product of the transition matrices. This equation can be solved approximately for large $\lambda$. In this manner asymptotic expansions for large eigenvalues can be calculated.

\section{BIBLIOGRAPHY ${ }^{2}$}

G. D. Birkhoff [1933], Quantum mechanics and asymptotic series, Bull. Amer. Math. Soc. 32, 681-700.

E. T. Copson [1965], Asymptotic expansions, Cambridge tracts in Mathematics and Mathematical Physics, no. 55, Cambridge Univ. Press, New York.

2 This list does in no way aim at completeness. More references-but also not a complete bibliography-for the literature before 1965 can be found in my book (Wasow [1965]). 
M. A. Evgrafov, and M. B. Fedoryuk [1966], Asymptotic behavior of solutions of the equation $w^{\prime \prime}(z)-p(z, \lambda) w(z)=0$ as $\lambda \rightarrow \infty$ in the complex $z$-plane, Uspehi Mat. Nauk 21, no. 1 (127) 3-50. (Russian)

W. B. Ford [1936], The asymptotic developments of functions defined by MacLaurin series, Univ. of Michigan Science Series, no. 11.

K. O. Friedrichs [1955], Asymptotic phenomena in mathematical physics, Bull. Amer. Math. Soc. 61, 485-504.

Nan Fröman, and P. O. Fröman [1965], JWKB approximation. Contributions to the theory, North-Holland, Amsterdam.

R. J. Hanson [1966], Reduction theorems for systems of ordinary differential equations with a turning point, J. Math. Anal. Appl. 16, 280-301. [1968], Simplification of second order systems of ordinary differential equations with a turning point, (to appear).

R. J. Hanson, and D. L. Russell [1967], Classification and reduction of second order systems at a turning point, J. Math. and Phys. 46, 74-92.

P.-F. Hsieh and Y. Sibuya [1966], On the asymptotic integration of second order linear ordinary differential equations with polynomial coefficients, J. Math. Anal. Appl. $16,84-103$.

H. K. Hughes [1945] The asymptotic developments of a class of entire functions, Bull. Amer. Math. Soc. 51, 456-461.

M. Iwano [1963] Asymptotic solutions of a system of linear ordinary differential equations containing a small parameter. I, Funkcial. Ekvac. 5, 71-134.

M. Iwano and Y. Sibuya [1963], Reduction of the order of a linear ordinary differential equation containing a small parameter, Ködai Math. Sem. Rep. 15, 1-28.

K. Kiyek [1967], Über eine spezielle Klasse linearer Differentialsysteme mit einem kleinen Parameter, Arch. Rational Mech. Anal. 25, 135-147.

R. Langer [1934], The asymptotic solutions of ordinary linear differential equations with special reference to the Stokes phenomenon, Bull. Amer. Math. Soc. 40, 545-582.

Roy Lee [1967], Asymptotic analysis of solutions of almost diagonal systems of ordinary differential equations at a turning point, Thesis, University of Wisconsin, Madison, Wis.

T. Nishimoto [1965a], On matching methods in turning point problems, Kōdai Math. Sem. Rep. 17, 198-221.

- [1965b], [1966], [1967], On matching methods for a linear ordinary differential equation containing a parameter. I, II, III, Kōdai Math. Sem. Rep. 17, 307-328; ibid., 18, 61-86; ibid., 19, 80-94.

T. Okubo [1966], On certain reduction theorems for systems of differential equations which contain a turning point, Proc. Japan Acad. 9, 544-549.

[1966], A global representation of a fundamental set of solutions and a Stokes phenomenon for a system of linear ordinary differential equations, J. Math. Soc. Japan 15, 268-288.

K. Okubo [1965], A connection problem involving a logarithmic function, Publ. Res. Inst. Math. Sci. Ser. A 1, 99-128.

F. W. J. Olver [1965], Error analysis of phase-integral methods. I: General theory for simple turning points; II. Applications to wave-penetration problems, J. Res. Nat. Bur. Standards Sect. B 69B, 271-290; ibid., 291-300.

Y. Sibuya [1962], Asymptotic solutions of a system of linear ordinary differential equations containing a parameter, Funkcial. Ekvac. 4, 83-113.

[1967], Subdominant solutions of the differential equation $y^{\prime \prime}-\lambda^{2}\left(x-a_{1}\right)$ $\left(x-a_{2}\right) \cdots\left(x-a_{m}\right) y=0$. 
G. Stengle [1961], A construction for solutions of an nth order linear differential equation in the neighborhood of a turning point, Ph.D. Thesis, Univ. Wisconsin, Madison, Wis.

[1964], Asymptotic solution of a class of second order differential equations containing a parameter, Report IMM-NYU 319, New York Univ., Courant Inst. of Math. Sciences, New York.

H. L. Turrittin [1950], Stokes multipliers for asymptotic solutions of a certain differential equation, Trans. Amer. Math. Soc. 68, 304-329.

W. Wasow [1961], Turning point problems for systems of linear equations. I: The formal theory, Comm. Pure Appl. Math. 14, 657-673.

[1962], Turning point problems for systems of linear differential equations.

II: The analytic theory, Comm. Pure Appl. Math. 15, 173-187.

[1963], Simplification of turning point problems for systems of linear differential equations, Trans. Amer. Math. Soc. 106, 100-114.

- [1965], Asymptotic expansions for ordinary differential equations, Pure and Appl. Math., vol. 14, Interscience, New York.

[1966a], On turning point problems for systems with almost diagonal coeficient matrix, Funkcial. Ekvac. 8, 143-171.

- [1966b], [1967], On the analytic validity of formal simplifications of linear differential equations. I, Funkcial. Ekvac. 9, 83-91; II, Funkcial. Ekvac. 10, 107-122. 